\title{
Análisis psicométrico del test de Fagerström de dependencia a la nicotina en una muestra de estudiantes universitarios de Arequipa, Perú
}

\author{
Psychometric analysis of the Fagerström test for \\ nicotine dependence in a sample comprising college \\ students in Arequipa, Peru
}

Correspondencia Walter L. Arias Gallegos warias@ucsp.edu.pe

Recibido: 07/06/2018

Arbitrado por pares

Aprobado: 29/08/2018

Citar como: Arias-Gallegos WL, Huamani-Cahua JC, Choque-Vera R. Análisis psicométrico del test de Fagerström de dependencia a la nicotina en una muestra de estudiantes universitarios de Arequipa, Perú. Acta Med Peru. 2018;35(3):174-9
Walter L. Arias-Gallegos ${ }^{1, a}$, Julio C. Huamani-Cahua ${ }^{1, a}$, Ronald Choque-Vera ${ }^{1, b}$

1 Universidad Católica San Pablo. Arequipa, Perú

a Doctor en Psicología, b Licenciado en Psicología

\section{RESUMEN}

Objetivos: Valorar las propiedades psicométricas del test de Fagerström de dependencia a la nicotina en una muestra de estudiantes universitarios de la ciudad de Arequipa. Materiales y métodos: Se trabajó con una muestra de estudiantes universitarios de una universidad privada de Arequipa. Todos participaron de manera voluntaria y fueron seleccionados mediante la técnica de muestreo por cuotas. El tamaño de la muestra fue se calculó mediante métodos probabilísticos con un nivel de confianza de $95 \%$, de manera estratificada. Se aplicó el test de Fagerström de dependencia a la nicotina en su versión de seis ítems. Resultados: Participaron 464 estudiantes, el 59,48\% eran mujeres y la edad media fue de $20 \pm 2,29$ años. Se pudo determinar la estructura unidimensional del instrumento con índices de bondad de ajuste adecuados a través del análisis factorial confirmatorio. La confiabilidad de la prueba fue 0,86 con la prueba Alfa de Cronbach y de 0,65 con la prueba Omega de McDonald. Conclusión: Se concluye que la prueba cumple con criterios aceptables de validez y confiabilidad y permite identificar a los estudiantes dependientes a la nicotina con puntuaciones iguales o superiores a 2,5 .

Palabras clave: Dependencia a la nicotina; Validez del test; Confiabilidad de los resultados (fuente: DeCS BIREME). 


\begin{abstract}
Objectives: To assess the psychometric capability of the Fagerström test for nicotine dependence in a sample comprising college students in Arequipa. Materials and methods: We worked using a sample comprising students enrolled in a private college in Arequipa. All subjects participated in a voluntary fashion, and they were selected using a quota sampling technique. The sample size was calculated using probabilistic methods and a $95 \%$ confidence level. The six-item version of the Fagerström test for nicotine dependence was administered. Results: Four-hundred and sixty-four students participated, $59.48 \%$ wee female, and the mean age was $20 \pm 2.29$ years. The one-dimensional nature of the instrument was determined using adjusted goodness of fit indexes which were set in place with a confirmatory factorial analysis. Reliability of the test was 0.86 according to Cronbach's alpha test, and it was 0.65 using Mc Donald's Omega test. Conclusion: The test complies with acceptable validity and reliability criteria, and it allows to identify students with dependence to nicotine, particularly those who achieved 2.5 or higher scores.
\end{abstract}

Keywords: Dependence, nicotine; Validity of tests; Reliability of results (source: MeSH NLM).

\section{INTRODUCCIÓN}

El consumo de tabaco es hoy en día un problema de salud global ya que constituye la primera causa de mortalidad y morbilidad, causando 5 millones de muertes por año (13500 muertes diarias) en todo el mundo ${ }^{[1]}$. El hábito de fumar se ha asociado con el desarrollo de cáncer en pulmón, cavidad oral, vejiga, esófago, estómago, páncreas, de mama y células sanguíneas ${ }^{[2]}$. Además, el consumo de cigarros acarrea una serie de problemas sociales pues afecta la economía familiar y acarrea gastos derivados del tratamiento de enfermedades asociadas ${ }^{[3]}$.

Asimismo, resulta preocupante que exista un incremento en el consumo de cigarrillos por parte de mujeres, adolescentes y niños ${ }^{[4]}$. Tal es así que, en el Perú, la edad de inicio de consumo sea a los 12 años, independientemente del sexo ${ }^{[5]}$. Adicionalmente, el $58 \%$ de la población ha consumido tabaco al menos una vez en su vida, el $34.7 \%$ al menos una vez en el último año, el $18,4 \%$ en el último mes y el $9,35 \%$ consumía tabaco de manera habitual[ ${ }^{[6]}$. En Argentina, se reportó una prevalencia de tabaquismo del $35 \%{ }^{[1]}$, en Colombia el $18 \%$ de adolescentes entre 13 y 18 años consumió tabaco al menos una vez en su vida ${ }^{[7]}$ y en España la prevalencia de consumo estuvo cerca del $30 \%{ }^{[4]}$.

Entre los factores que inciden en su consumo y habituación están los culturales, que comprenden la promoción del consumo a través de los medios de comunicación y el fácil acceso a estas sustancias que son social y legalmente aceptadas ${ }^{[1]}$; biológicos, explicados por los receptores nicotínicos y la porción lateral del hipotálamo (más específicamente el fascículo prosencefálico mediano) que juegan un papel crucial en el reforzamiento de las conductas adictivas ${ }^{[8]}$; y psicológicos, que a través de los procesos de condicionamiento clásico, operante y vicario permiten explicar el mantenimiento de tales conductas ${ }^{[9]}$.

Todo ello ha generado la necesidad de valorar los hábitos de consumo de tabaco en la población, a través de diseños de investigación transversal y longitudinal. En el primer caso, América Latina, Brasil, Argentina y México son los países con mayor cantidad de estudios epidemiológicos sobre el tabaquismo, reportándose que los varones de clase social baja son quienes tienen mayor prevalencia de consumo, por lo que se presume que el estrés psicosocial es un factor determinante ${ }^{[3]}$. En ese sentido, en un estudio realizado en Arequipa, Perú se encontró que el estrés laboral se asociaba con un mayor consumo de bebidas alcohólicas, pero no de cigarrillos ${ }^{[10]}$; además, entre los estudiantes universitarios, la prevalencia de consumo es muy baja (5\%) y tiene un impacto negativo en su rendimiento académico ${ }^{[11]}$. En cuanto a los estudios longitudinales, se reporta un ligero decremento en el consumo de cigarros en los últimos años y cambios en las características de fumar: una menor dependencia a la nicotina y menor porcentaje de fumadores diarios, aunque los fumadores ocasionales aumentaron ${ }^{[4]}$.

Un problema para investigar el tabaquismo radica en la gran diversidad metodológica en que se sustentan los estudios y la variedad de instrumentos para su valoración. Aunque los criterios bases se fundamentan en el DSM como la prioridad, la tolerancia, la continuidad y la estereotipia; las pruebas que evalúan la dependencia a la nicotina o el tabaquismo tienen criterios de corte diferentes y persiguen objetivos igualmente diferentes ${ }^{[12]}$.

En tal sentido, si bien la conducta de fumar puede ser evaluada a través de distintos métodos las entrevistas, pruebas médicas, cuestionarios de autorregistro, pruebas psicométricas y medidas fisiológicas ${ }^{[13]}$; las pruebas psicológicas son las más usadas para el tamizaje y fundamentación de la valoración diagnóstica. Así pues, existe una gran variedad de pruebas diseñadas para la valoración de la conducta de fumar, entre las que se tiene el Smoking Withdrawal Questionnaire, el Smoker Complaint Scal, el Wisconsin Smoking Withdrawal, el Minnesota Smoking Withdrawal Scale, el Smoking Consequences Questionnaire, la Escala de autoeficicacia para evitar de fumar de Diclemente, el Cuestionario de seguimiento de abstinencia y recaída, la Escala de evaluación del síndrome de dependencia a la nicotina, el test de Fagerström de dependencia de la nicotina, etc. ${ }^{[13]}$. 
El test de Fagerström ha sido el más usado a nivel mundial y ha servido de base para la elaboración de otros instrumentos como el Cuestionario para la clasificación de consumidores de cigarrillo ${ }^{[7]} \mathrm{y}$ como medida de comparación para obtener la validez concurrente de otros instrumentos como la Escala de evaluación del síndrome de dependencia a la nicotina ${ }^{[14]}$. Fue creado en 1978 con la finalidad de discriminar a los fumadores eventuales de los dependientes a la nicotina ${ }^{[15]}$. Tiene dos versiones, la primera de ocho ítems y la segunda de seis. En ambos casos se considera que la nicotina es un reforzador primario de la conducta adictiva hacia el consumo de cigarrillos y evalúa el número de cigarrillos consumidos por día, la dosis de nicotina que se consume, el tiempo que se demora en fumar después de levantarse y la necesidad de fumar en lugares y situaciones en que no es debido. La versión de seis ítems es más reciente y aunque evalúa aspectos similares el punto de corte suele ser seis, a diferencia de la primera escala que es de $11^{[16]}$.

Esta prueba ha sido utilizada en los países de habla hispana y ha sido sometida a diversas valoraciones psicométricas para verificar sus propiedades diagnósticas ${ }^{[17]}$. Sin embargo, en la ciudad de Arequipa no hay estudios psicométricos con este instrumento, a pesar de ser usado como una prueba de tamizaje en el Ministerio de Salud del Perú (MINSA) lo que ha motivado el presente estudio. De este modo, el fin de la presente investigación es analizar la validez y confiabilidad del test de Fagerström de dependencia de la nicotina en una muestra de estudiantes universitarios arequipeños.

Cabe señalar que, en reportes previos, se ha visto que presenta validez concurrente con otros instrumentos ${ }^{[7,14]}$, y que Becoña ${ }^{[17]}$ reportó una estructura unidimensional y niveles moderados de confiabilidad. Aunque otros reportes más recientes señalan que, si bien el Test de Fagerström es confiable $(0,81)$, otras pruebas como la Escala de evaluación del síndrome de dependencia a la nicotina tienen niveles de consistencia interna ligeramente mayores $(0,86)^{[18]}$.

\section{MATERIALES Y MÉTODOS}

\section{Diseño y población del estudio}

Se trata de una investigación de tipo tecnológica ${ }^{[19]}$ con un diseño de investigación instrumental ${ }^{[20]}$. La muestra estuvo conformada por estudiantes de una universidad privada de la ciudad de Arequipa de ambos sexos. Para la selección de la muestra se realizó un muestreo probabilístico con el $95 \%$ de confianza y un $5 \%$ de margen de error, y se estratificaron submuestras de acuerdo al tipo de carrera profesional.

\section{Instrumento}

Se utilizó la versión de seis ítems del test de Fagerström de dependencia de la nicotina, de los cuales cuatro tienen una respuesta dicotómica y dos respuestas tipo Likert de 0 a 3 . El puntaje puede variar de 0 a 10 puntos ${ }^{[16]}$. Se valoró el momento y la frecuencia del consumo de cigarrillos así como la dificultad para dejar de fumar. Se consideró como punto de corte para diferenciar entre fumadores dependientes y no dependientes de la nicotina el valor de 6 puntos ${ }^{[13]}$.

\section{Procedimientos y análisis estadístico}

La evaluación se realizó en las áreas comunes de la Universidad. Luego de obtener el consentimiento informado, se aplicó el instrumento. Posteriormente, se realizó el modelo factorial confirmatorio (AFC) con la librería Lavaan de $R$, usándose el estimador WLSMV (mínimos cuadrados ponderados y con media y varianza ajustada) ${ }^{[21]}$. Es decir, se utilizó, el método de mínimos cuadrados no ponderados robustos para variables policóricas con datos no específicos sin errores correlacionados.

Este estimador presenta robustez en los resultados en situaciones de no normalidad y escala ordinal. Para confirmar el modelo se tuvo en cuenta el índice de ajuste comparativo (CFI), con valores $\geq 0,90^{[22]}$. La raíz residual estandarizada cuadrática media (SRMR) y el error cuadrático medio de aproximación (RMSEA), con valores $\leq 0,08^{[23]}$, valores que evidencian ajuste al modelo.

Para el análisis de confiabilidad se usó el estadístico denominado omega de Mc Donald. Este coeficiente es una alternativa ante las limitaciones discutidas del coeficiente alfa de Cronbach ${ }^{[24]}$. Asimismo, la precisión diagnóstica del test de Fagerström de dependencia de la nicotina se midió a través de la curva ROC (Receiver Operating Characteristic curve - ROC curve).

\section{Aspectos éticos}

Para poder participar en el estudio debió firmar un consentimiento informado que incluía la explicación de los fines del estudio y su aceptación expresa a participar de la investigación. Se les garantizó la reserva de sus datos personales y la confidencialidad de la información. La investigación fue aprobada por el Comité de ética.

\section{RESULTADOS}

La muestra estuvo conformada por 464 estudiantes. Respecto a la carrera profesional, el 5,60\% pertenecía a Ingeniería electrónica y telecomunicaciones, 5,38\% a Ciencias de la computación, 11,85\% a Psicología, 22,19\% a Ingeniería Industrial, 19,61\% a Administración de negocios, 4,31\% a Educación, 15,08\% a Derecho, 4,95\% a Ingeniería civil y 10,99\% a Contabilidad.

El 59,48\% de los participantes fueron mujeres. La edad promedio fue de $20 \pm 2,29$ años, dentro de un rango de 17 y 31 años.

En la Tabla 1 se presenta el análisis descriptivo de los ítems, encontrando medias aritméticas altas y dispersas en los ítems 1 y 3 . Los estadísticos descriptivos muestran que los ítems 1 y 4 presentan valores elevados de asimetría y curtosis, considerando que los valores dentro del umbral $\pm 1,5$ indican variaciones dentro 
de la normalidad ${ }^{[25]}$ por lo que las distribuciones no son normales. En ese sentido, se utilizó el estimador WLSMV (mínimos cuadrados ponderados y con media y varianza ajustada) que presenta robustez en los resultados en situaciones de no normalidad.

Tabla 1. Análisis de los ítems del test de Fagerström de dependencia de la nicotina

\begin{tabular}{ccccc} 
Pregunta & $\mathbf{x}(\mathbf{I C}$ 95\%) & $\boldsymbol{\sigma}$ & $\mathbf{g}_{1}$ & $\mathbf{g}_{2}$ \\
\hline Ítem 1 & $0,06(0,022-0,094)$ & 0,39 & 6,960 & 47,936 \\
Ítem 2 & $0,15(0,121-0,186)$ & 0,36 & 1,934 & 1,748 \\
Ítem 3 & $0,16(0,123-0,191)$ & 0,37 & 2,016 & 2,511 \\
Ítem 4 & $0,03(0,011-0,045)$ & 0,19 & 7,514 & 61,854 \\
Ítem 5 & $0,08(0,053-0,102)$ & 0,27 & 3,168 & 8,073 \\
Ítem 6 & $0,08(0,051-0,099)$ & 0,26 & 3,226 & 8,442 \\
\hline
\end{tabular}

$\mathrm{n}=2470$

x: Media; IC 95\%: intervalo de confianza al 95\%; : varianza; g1: asimetría; g2: curtosis

La Tabla 2 muestra la matriz de correlación para evaluar la fuerza y dirección de la relación entre los ítems, observando que los ítems no están altamente correlacionados, entonces, los elementos podrían medir diferentes características o no estar claramente definidos.

Tabla 2. Matriz de correlaciones de los ítems del test de Fagerström de dependencia de la nicotina.

Pregunta Ítem 1 Ítem 2 Ítem 3 Ítem 4 Ítem 5 Ítem 6

\begin{tabular}{lcccccc} 
Ítem 1 & 1 & -- & -- & -- & -- & -- \\
Ítem 2 & $0,181^{*}$ & 1 & -- & -- & -- & -- \\
Ítem 3 & $0,204^{*}$ & $0,272^{*}$ & 1 & -- & -- & -- \\
Ítem 4 & $0,355^{*}$ & $0,127^{*}$ & $0,183^{*}$ & 1 & -- & -- \\
Ítem 5 & $0,121^{*}$ & $0,212^{*}$ & $0,182^{*}$ & $0,297^{*}$ & 1 & -- \\
Ítem 6 & $0,124^{*}$ & $0,332^{*}$ & $0,187^{*}$ & $0,303^{*}$ & $0,314^{*}$ & 1 \\
\hline
\end{tabular}

* La correlación es significativa en el nivel 0,01 (bilateral).

La Tabla 3, muestra el análisis factorial confirmatorio para la estructura original de una dimensión conformada por seis ítems, el mismo que indica un ajuste robusto apropiado, $\chi^{2}(15)=$ $247,3, \mathrm{CFI}=986, \mathrm{RMSEA}=0,03, \mathrm{SMRM}=0,084$. En este análisis se utilizó un método de estimación robusto para el cálculo de $\chi^{2}$ y de los errores estándar debido a los indicios razonables de no multinormalidad entre los ítems.
La Tabla 4 muestra las cargas factoriales, que se encuentran entre 0,613 y 0,874 . La versión estandarizada de los coeficientes, equivale a un coeficiente de regresión parcial en la cual se han extraído el efecto que otras variables puedan tener en esta relación. Como se observa en esta tabla, las saturaciones son altas, lo que lleva a afirmar con certeza que la estructura jerárquica del instrumento y las magnitudes observadas en las saturaciones se ajustan bien a las respuestas de los participantes al test.

Tabla 4. Cargas factoriales de la solución estandarizada del AFC para el modelo final.

\begin{tabular}{lc}
\multicolumn{1}{c}{ Ítems } & F1 \\
$\begin{array}{lc}\text { 1. ¿Cuánto tiempo pasa entre que se levanta y } \\
\text { fuma su primer cigarrillo? }\end{array}$ & 0,740 \\
$\begin{array}{l}\text { 2. ¿Encuentra difícil no fumar en lugares donde } \\
\text { está prohibido? }\end{array}$ & 0,715 \\
$\begin{array}{l}\text { 3. ¿Qué cigarrillo le molesta más dejar de fumar? } \\
\text { 4. ¿Cuántos cigarrillos fuma cada día? }\end{array}$ & 0,613 \\
$\begin{array}{l}\text { 5. ¿Fuma con más frecuencia durante las } \\
\text { primeras horas después de levantarse que }\end{array}$ & 0,874 \\
$\quad \begin{array}{l}\text { durante el resto del día? } \\
\text { 6. ¿Fuma, aunque esté enfermo y tenga que } \\
\text { guardar cama la mayor parte del día? }\end{array}$ & 0,795 \\
\hline
\end{tabular}

La confiabilidad se vincula con el error aleatorio, en donde a mayor error aleatorio, menor confiabilidad ${ }^{[26]}$. Para considerar un valor aceptable de confiabilidad mediante el coeficiente omega, este debe encontrarse entre 0,70 y $0,90^{[27]}$, aunque en algunas circunstancias pueden aceptarse valores superiores a $0,65^{[28]}$. En ese sentido, la consistencia interna para los seis ítems del Test de Fagerström muestra un Alfa de Cronbach de 0,86 y el Omega de McDonald de 0,65.

Tabla 5. Valores para la curva ROC de la variable dependencia a la nicotina.

\begin{tabular}{cccc}
\hline Criterio & Sensibilidad & Especificidad & $\begin{array}{c}\text { Índice de } \\
\text { Youden }\end{array}$ \\
\hline$-1,00$ & 1,000 & 1,000 & 0,000 \\
0,50 & 1,000 & 0,265 & 0,735 \\
1,50 & 1,000 & 0,073 & 0,927 \\
2,50 & 1,000 & 0,000 & 1,000 \\
3,50 & 0,519 & 0,000 & 0,519 \\
4,50 & 0,185 & 0,000 & 0,185 \\
6,00 & 0,111 & 0,000 & 0,111 \\
8,00 & 0,037 & 0,000 & 0,037 \\
10,00 & 0,000 & 0,000 & 0,000 \\
\hline
\end{tabular}

Tabla 3. Índices de bondad de ajuste del Test de Fagerstrom.

\begin{tabular}{cccccccccc} 
Modelo & \multirow{2}{*}{$\chi^{2}$} & gl & CFI & RMSEA & Satorra- & CFI & RMSEA & SMRM \\
\cline { 3 - 10 } & & & & Bentler $\chi^{2}$ & Robusto & Robusto & Robusto \\
\hline Modelo 1 & 418,7 & 15 & 1 & $0,00(0,00,0,05)$ & 274,3 & 0,986 & $0,03(0,00,0,06)$ & 0,084 \\
\hline
\end{tabular}

CFI: Índice de ajuste comparativo; RMSEA: error cuadrático medio de aproximación; SMRM: raíz residual estandarizada cuadrática media, p<0,001 
Para hallar el punto de corte óptimo se utilizó la curva de ROC; en este caso se analizó la variable de prueba (puntaje total del Test de Fagerström). Para este procedimiento se necesita una evaluación de referencia dicotómica (Gold Standard) que identifique en sujeto dependiente/no dependiente a la nicotina, es decir, puntos de corte preestablecidos, para lo cual, se tomaron en cuenta dos referencias, el primero $2 / 10^{[29]}$, y punto de corte $4 / 10^{[30]}$. Sin embargo, atendiendo al tamaño de la muestra y poder tener una ratio de $1 / 10$ o $1 / 20$ se consideró el punto de corte coprológico de 2/10, observando, 27 estudiantes universitarios dependientes a la nicotina y 437 no dependientes.

En ese sentido el punto de corte óptimo fue de 2,5 (Tabla 5), que interpreta a la sensibilidad 1.000 como la probabilidad del $100 \%$ de clasificar correctamente a un sujeto dependiente a la nicotina y la especificidad como la probabilidad de clasificar correctamente a un sujeto como no dependiente a la nicotina (para ello se utilizó el índice de Youden ${ }^{[31]}$, que optimizó el punto de corte). Asimismo, el punto $(0,1)$, para sensibilidad y especificidad, representa la clasificación perfecta, totalidad de verdaderos positivos, y ningún falso positivo ${ }^{[32]}$.

\section{DISCUSIÓN}

El presente estudio tuvo por finalidad la valoración de las propiedades psicométricas del test de Fargerström de dependencia de la nicotina en una muestra de estudiantes universitarios de la ciudad de Arequipa con una edad media de 20 años, que se ubica dentro del rango de edad con mayor predisposición al consumo de cigarrillos ${ }^{[5]}$, ya que diversos estudios han puesto de manifiesto que los universitarios suelen recurrir al consumo de sustancias psicoactivas como una estrategia de afrontamiento del estrés ${ }^{[33]}$.

Asimismo, el Test de Fagerström ya es usado como una prueba de tamizaje por el MINSA, sin embargo, no existen reportes sobre sus medidas de validez y confiabilidad. Nuestros resultados evidencian que dicho instrumento es válido para la muestra de universitarios de Arequipa al contar con una estructura factorial con índices de bondad de ajuste adecuados para una sola dimensión como ha sido previsto por el autor ${ }^{[16]}$, y que se ha verificado en otros estudios con muestras hispanoparlantes ${ }^{[17,18]}$.

Con respecto a la confiabilidad, se ha obtenido un valor de 0,86 con el método de consistencia interna y la prueba Alfa de Cronbach, que es consistente otros reportes ${ }^{[18]}$; pero al calcular la confiabilidad con la prueba Omega de McDonald el índice se reduce a 0,65 como se ha reportado en otros estudios ${ }^{[17]}$. Esto supone tener cautela a la hora de valorar los datos del test de Fagerström porque presenta una consistencia sensible a ciertas variantes psicométricas.

Por otro lado, el punto de corte de la prueba se determinó por medio de las propiedades de sensibilidad y especificidad de las curvas ROC, y se encontró que el valor de 2,5 fue el más adecuado para discriminar entre los sujetos dependientes de los no dependientes a la nicotina. En ese sentido, otros estudios reportaron que el punto de corte adecuado sería el valor de $6^{[12]}$, pero también se suele tomar como criterio la cantidad de cigarros que se fuma. Así, Becoña ${ }^{[17]}$ ha propuesto que para los españoles fumar de 1 a 15 cigarrillos diarios sería indicador de un fumador leve, de 16 a 30 fumador normal y de 31 a más de un fumador severo. Londoño et al. ${ }^{[7]}$ propusieron por su parte, que, para la población colombiana, un fumador leve consume cinco o menos cigarrillos diarios, un fumador moderado entre 6 y 15 cigarrillos diarios y un fumador severo de 16 o más cigarrillos por día. Datos que son consistentes con los reportes de la Organización Mundial de la Salud.

Bajo los criterios propuestos se pudo determinar que 27 estudiantes $(5,81 \%)$ cumplían con los criterios diagnósticos de dependencia a la nicotina, cifra que se aproxima con la de un estudio local que determinó que la dependencia a la nicotina afectaba al $1 \%$ de la población joven de la región ${ }^{[11]}$. Nuevos estudios, sin embargo, deberán superar las limitaciones de la presente investigación, como son la falta de representatividad de la muestra con respecto a otras instituciones de nivel superior de la localidad y la necesidad de obtener datos sobre la validez concurrente, divergente y predictiva.

Finalmente, podemos concluir que el test de Fargerström de dependencia de la nicotina cuenta con adecuados índices de validez y confiabilidad para la muestra de estudiantes universitarios de la ciudad de Arequipa, por lo que se sugiere su uso en muestras similares. Por otro lado, cabe recalcar que es necesario realizar más investigaciones sobre diversos temas relacionados con el consumo de cigarrillos y la dependencia a la nicotina como la disposición a dejar de fumar ${ }^{[34]}$, la relación entre personalidad y consumo de sustancias psicoactivas y otros trastornos psicológicos, o el nivel de tabaquismo en sujetos dependientes con respecto a la etapa de craving ${ }^{[13]}$. Además, es importante fortalecer los valores familiares, pues las variables familiares han tenido un efecto preventivo y protector sobre el consumo de sustancias psicoactivas en los adolescentes y jóvenes ${ }^{[35]}$.

Fuente de financiamiento: Los autores declaran no haber recibido ningún tipo de financiamiento para la realización del presente trabajo de investigación

Declaración de conflicto de intereses: Los autores declaran no tener conflicto de intereses con la publicación del artículo.

\section{REFERENCIAS BIBLIOGRÁFICAS}

1. Novoa-Gómez MM, Barreto I, Silva LM. Consumo de cigarrillo y prácticas culturales en contextos universitarios. Rev Latinoam Psicol. 2012;44(1):97-110.

2. Rojas C (Ed.). Drogas. Conceptos, miradas y experiencias. Chile: Universidad Católica del Maule; 2015.

3. Bardach A, García HA, Ruano RA, Ciapponi A. Niveles de ingreso y prevalencia de tabaquismo en América Latina: revisión sistemática y metaanálisis. Rev Panam Salud Publica. 2016;40(4):263-71.

4. Miguez MC, Becoña E. El consumo de tabaco en estudiantes de Psicología a lo largo de 10 años (1996-2006). Psicothema. 2009;21(4):573-8. 
5. Instituto Apoyo. Informe sobre la situación del consumo de tabaco en menores de edad y programas de prevención de fumar en menores en el Perú. Lima: Apoyo; 2004.

6. Naciones Unidas. Drogas y delitos en Perú. Situación actual y evolución. Lima: UN; 2008.

7. Londoño C, Rodríguez I, Gantiva CA. Cuestionario para la clasificación de consumidores de cigarrillo (C4) para jóvenes. Divers Perspect Psicol. 2011;7(2):281-91.

8. Arroyo G, Aldea M, Fuentealba J, García AG. Receptor nicotínico, galantamina y enfermedad de Alzheimer. Rev Neurol. 2002;34(11):1057-65.

9. Arias WL. Fundamentos del aprendizaje. Arequipa: Vicarte; 2008.

10. Arias WL. Estrés laboral y consumo de sustancias psicoactivas (SPA) desde un enfoque de la salud ocupacional. Rev Per Psic Trab Soc. 2012;1(1):107-18

11. Choque R, Arias WL. Relación del consume de cigarrillos con el rendimiento académico en estudiantes universitarios. PsiqueMag. 2017;6(1):151-63.

12. Becoña E, Fernández E, López A, Míguez MC, Castro J, Nogueiras L, et al. La escala breve de evaluación del Síndrome de Dependencia de la Nicotina (NDSS-S) en fumadores españoles. Psicothema. 2011;23(1):126-32.

13. Becoña E, Lorenzo MC. Evaluación de la conducta de fumar. Adicciones. 2004;16(2):201-26.

14. Becoña E, Nogueiras L, Flórez G, Álvarez S, Vázquez D. Propiedades psicométricas de la escala de Síndrome de Dependencia de la Nicotina (NDSS) en una muestra de fumadores que solicitan tratamiento por su dependencia del alcohol. Adicciones. 2010;22(1):37-50.

15. Fagerström KO. Measuring degree of physical dependence on tobacco smoking with references to individualization of treatment. Addict Behav. 1978;3(3-4):235-41.

16. Heatherton TF, Kozlowski LT, Frecker RC, Fargerström KO. The Fagerström Test for Nicotine Dependence: a revision of Fagerström Tolerance Questionnaire. Br J Addict. 1991;86(9):1119-27.

17. Becoña $E$, Vázquez $F$. The Fagerström test for nicotine dependence in a Spanish simple. Psychol Rep. 1998;83(3 Pt 2):1455-8.

18. Becoña $E$, Fernández E, López A, Míguez MC. La escala de Síndrome de Dependencia de la Nicotina (NDSS) en una muestra de fumadores que demandan tratamiento para dejar de fumar. Psicothema. 2009;21(4):579-84.

19. Sánchez $H$, Reyes C. Diseño y metodología de la investigación. Lima: Universidad Ricardo Palma; 2014.
20. Ato $\mathrm{M}$, López JJ, Benavente A. Un sistema de clasificación de los diseños de investigación en psicología. Anal Psicol. 2013;29(3):103859.

21. Kline R. Principles and practice of structural equation modelling (4ta ed.). New York: The Guilford Press; 2015.

22. Bentler P. Comparative fit indices in structural models. Psych Bull. 1990;107(2):238-46.

23. MacCallum RC, Browne MW, Sugawara HM. Power analysis and determination of sample size for covariance structure modeling of fit involving a particular measure of model. Psychol Methods. 1996; 13(2): 130-49.

24. Cho E. Making reliability reliable: A systematic approach to reliability coefficients. Organizational Research Methods. 2016;19(4):651-82.

25. George D, Mallery MP. SPSS for Windows step by step: A simple guide and reference. Boston, MA: Allyn \& Bacon; 2001.

26. Kupermintz H. On the reliability of categorically scored examinations. J Educ Meas. 2004;41(3):193-204.

27. Campo-Arias A, Oviedo HC. Propiedades psicométricas de una escala: la consistencia interna. Rev Salud Pública. 2008;10(5):831-9.

28. Katz MH. Multivariable analysis (2a ed.). Cambridge: Cambridge University Press; 2006.

29. Campo-Arias A, Herazo E, Barros J, Rueda G, Díaz L, Díaz F. Dependencia a la nicotina: desempeño psicométrico de dos escalas en adultos. Investig Andina. 2011;13(23):258-67.

30. Mataix J. Propiedades psicométricas del test de Fagerström con dos preguntas reformuladas. (Tesis Doctoral). Alicante, España: Universidad Miguel Hernández; 2011.

31. Youden, W. J. Index for rating diagnostic tests. Cancer. 1950;3(1):32-

32. Estrada J. El índice de Youden y su aplicación a la determinación de punto de corte en un test cuantitativo (Tesis de maestría). Granada, España: Universidad de Granada; 2016.

33. Chau C. Factores psicosociales asociados el uso de bebidas alcohólicas y tabaco en adolescentes tardíos de una universidad privada de Lima. Rev Psicol (Lima). 2005;13(2):161-91.

34. Roth E, Exeni S. Exploración de la validez y la confiabilidad de Instrumento de la Medición de la Disposición al Cambio en fumadores habituales. Ajayu. 2010;8(2):101-21.

35. Martínez JM, Robles L. Variables de protección ante el consumo de alcohol y tabaco en adolescentes. Psicothema. 2001;13(2):222-8. 